\section{Surgical treatment of necrotic scleral calcification using combined conjunctival autografting and an amniotic membrane inlay filling technique}

B-H Kim

Conclusion The combined technique had high success rates of graft survival and good revitalization of the necrotic area of scleral calcification, eliminated the need for invasive and time-consuming scleral autografting or allografting, and provided good cosmesis. Scleral ischaemia, which was caused by MMC, may induce scleral calcification.

Eye (2011) 25, 1484-1490; doi:10.1038/eye.2011.209; published online 26 August 2011

Keywords: scleral calcification; conjunctival autograft; amniotic membrane inlay filling technique; pterygium; regional conjunctivectomy; mitomycin C

\section{Introduction}

Pterygium surgery with adjuvant mitomycin C (MMC) is associated with development of scleral calcification, ${ }^{1-3}$ the exact mechanism of which is unknown. Even though scleral calcification is not a common complication, it produces an overlying conjunctival defect, which may allow access to pathogens. ${ }^{4}$ Once an infection is established, it is extremely difficult to control and may lead to infectious necrotising scleritis, which is the most devastating complication. Therefore, prevention of the catastrophic infection is more important than treatment.

To prevent the complication, the ocular surface should be reconstructed. One study reported successful treatment of scleral calcific plaques $(2 \times 1 \mathrm{~mm})$ by simple excision, and 
claimed that the underlying sclera was intact and the area healed uneventfully. ${ }^{3}$ In most cases, however, removal of the space-occupying lesion leaves a surface defect that requires substituting healthy vascularized tissue for degenerated calcified tissue. Various biological tissues including cartilage, ${ }^{5}$ fascia lata, ${ }^{6}$ dermis, ${ }^{5,6}$ pericardium, ${ }^{7,8}$ sclera, ${ }^{9,10}$ and cornea ${ }^{11-13}$ have been used to fill the defect. However, none is adequate if the defect is small, because the techniques are too invasive, complicated, and time-consuming to treat such a small lesion, and the substrates are not easy to obtain. Teping and Reim ${ }^{14}$ reported a technique, known as Tenonplasty, to treat scleral melt in patients with severe chemical and thermal ocular burns. Tenonplasty was successfully used in conjunction with lamellar corneal patch graft and amniotic membrane transplantation for removing calcified plaques and reconstructing the ocular surface. ${ }^{15}$ However, this technique was used when patients had calcified plaques associated with extensive areas of ischaemia, severe scleral melt, and/or uveal exposure. Conjunctival transplantation alone also has been reported to treat scleral melt. ${ }^{16,17}$ However, those cases were not associated with scleral calcification, but with surgically induced necrotising scleritis.

Regional conjunctivectomy was developed to treat chronic conjunctival hyperaemia. ${ }^{18}$ The technique removes the conjunctiva and Tenon's capsule from the limbus to near the plica semilunaris medially, and from the limbus to beside the lateral canthus laterally. Among patients who had undergone this technique and application of postoperative MMC previously for treating pterygia or pingueculae, scleral calcification was identified.

I developed a new surgical technique to treat the early stage of scleral calcification, which precedes the development of scleral melt and other serious complications by removing the calcific plaque safely, and preserving the scleral thickness using combined conjunctival autografting and amniotic membrane transplantation. Amnion was used to fill the space that had been occupied by the calcific plaque. Lee and Tseng $^{19}$ reported the effectiveness of amniotic membrane as a filling material for treating deep corneal ulcers and to protect the graft. In the current study, I describe the surgical technique and report on its clinical usefulness.

\section{Materials and methods}

The current study was an interventional case series of 10 patients (11 eyes and 12 regions) who presented to the Seer and Partner Eye Institute, Seoul, Republic of Korea. All patients had previously undergone regional conjunctivectomy with postoperative MMC to treat pterygia ( 2 patients) or pingueculae ( 8 patients) at my institution. Nine patients had no specific past ocular history at the time of regional conjunctivectomy; one had a history of laser vision correction surgery. Calcific plaques, located in the centre of avascular areas of the sclera, were identified incidentally during follow-up after previous surgeries. Surgery was indicated if the calcific plaques progressively increased in size or if there was a defect on the overlying conjunctiva.

All patients provided informed consent preoperatively. The same surgeon (B-HK) performed all surgeries. Five sets of proparacaine hydrochloride $0.5 \%$ drops (Alcaine, Alcon, Fort Worth, TX, USA) were administered for topical anaesthesia. A bridle suture with no. 6-0 black silk was placed at the 9 or 3 o'clock position on the limbus, depending on whether the right or the left eye was undergoing surgery to allow as much access as possible to the surgical field. Calcific plaques were outlined and separated from the scleral side walls with a no. 15 Bard-Parker blade. Using a bevel-down disposable crescent knife (Sharpoint Sharptome, Angoitech Pharmaceuticals Inc., Vancouver, Canada), the calcific plaque was undermined, with care taken to avoid damaging the underlying sclera. When the undermining was completed, the scleral plaque was lifted and removed. The scleral bed was cleaned with a spearshaped sponge. The size of the scleral defect was measured with a surgical caliper horizontally and vertically. The patients were instructed to look down to allow exposure of the superior bulbar area. The conjunctival area was marked for harvesting of a donor graft that was of the same size as the scleral defect. The conjunctival epithelial layer without the subconjunctival connective tissue was dissected using Wescott scissors. Bleeding points were electrocauterized. No sutures were used to close the donor site. The harvested conjunctival tissue was grafted on the scleral defect and secured with multiple no. 10-0 nylon-interrupted sutures; a small gap was left, through which amniotic membrane could be inserted. Suture knots were buried inside the sclera. A sheet of cryopreserved amniotic membrane was cut to $2 \mathrm{~mm}$ wide and as long as needed. The amniotic membrane was inserted randomly into the space between the conjunctival graft and the scleral bed, until the space was adequately filled. After confirming that the surfaces of the graft and the surrounding sclera were level, the small opening was closed with a no. 10-0 nylon suture. To protect the graft site, the amniotic membrane was transplanted over the grafted site, stromal side up, and fixed onto the underlying sclera using no. 10-0 nylon sutures. If the lid tension was considered sufficiently strong to damage the temporary protective amniotic membrane patch, a 19-mm oversized bandage contact lens (Ologen, Aeon Astron Corporation, Taipei City, 
Taiwan) was applied at the end of surgery to further protect the graft.

Postoperatively, tobramycin $0.3 \%$ eye drops (Ocuracin, Samil Pharmaceutical Company, Seoul, Republic of Korea) and fluorometholone $0.1 \%$ eye drops (Ocumetholone, Samil Pharmaceutical Company) were instilled twice daily for 3 weeks. Carboxymethylcellulose sodium $0.5 \%$ eye drops (Refresh Plus, Allergan, Irvine, CA, USA) were instilled frequently.

When the patients were examined 1 week postoperatively, the bandage contact lens was removed. The temporary amniotic membrane graft was removed 2 weeks postoperatively. The conjunctival graft sutures were removed if the surface epithelialization of the graft was completed. If the epithelialization was incomplete, the sutures were retained until the surface was healed completely. All applicable institutional and governmental regulations concerning the ethical use of human volunteers were followed during this research.

\section{Results}

Table 1 shows each patient's clinical characteristics. All calcific plaques were less than $4 \mathrm{~mm}$ in size, and most were between $2 \times 2$ and $2.5 \times 3 \mathrm{~mm}$. During the previous surgery, two eyes of two patients and nine eyes of eight patients were treated with $0.02 \% \mathrm{MMC}$ four times daily for 3 days and 5 days postoperatively, respectively. The mean time between development of scleral calcification and the previous surgery was 11.2 \pm 3.2 months (range, 5-17 months). Scleral calcification developed in 8 of 12 regions medially and 4 laterally. The calcific plaques developed in ischaemic areas of the sclera (11 of 12 regions). Figure 1 shows how the scleral calcification developed and evolved in case 1 .

Patients were followed for 7 to 18 months postoperatively (mean, $11.0 \pm 3.5$ months). Nine patients were treated successfully with one surgery, but one patient (case 10) needed another surgery because the graft from the first surgery, which was performed without the amniotic membrane inlay filling technique, did not survive. Epithelialization over the graft was completed by 2 weeks postoperatively, except in cases 3 and 10 . In case 3 , partial detachment of the transiently transplanted amniotic membrane, which was used to protect the surgical site, was identified 1 week postoperatively. Vascular growth into the graft from the surrounding tissue, which is essential for graft survival, occurred in all cases (Figure 2); in six cases, it occurred simultaneously with epithelialization, and in cases 2, 7, 9, and 10, the time exceeded 9 weeks. Even during the time without vascularization, the grafts survived.

\section{Discussion}

Scleral calcification must be treated for three reasons. First, it is not a physiological tissue in the sclera. Second, as the current study showed, the calcification tended to increase in size over time, which may lead to serious progressive scleral thinning or scleromalacia, because the calcification is a degenerative change. Third, the calcific plaque induces an overlying surface defect through which pathogens may establish infection. ${ }^{4}$ In the current study, half of the cases developed a surface defect. Even though no infections developed, a scleral defect over the long term is prone to invasion by microorganisms. It is important to treat the ocular surface as early as possible to prevent a devastating complication such as necrotising infectious scleritis.

In the current study, I used the conjunctiva and amniotic membrane as graft tissues. The conjunctival donor tissue is easy to harvest from the superior bulbar conjunctiva and provides abundant blood vessels that help to restore vascularization of the ischaemic areas and ensure graft survival. Using the amniotic membrane was helpful because it easily filled the space between the conjunctival autograft and the scleral bed, and made the ocular surface flat and regular. I refer to this procedure as the amniotic membrane inlay filling technique. Amniotic membrane also probably maintains the viability of the overlying conjunctival graft during the period of graft vascularization by providing hydration. In cases 2,7 , and 9, and in the second surgery in case 10, the conjunctival graft survived without any blood vessels for weeks until vascularization occurred. A conjunctival autograft without amniotic membrane inlay filling failed during the first surgery in case 10. The success of the second surgery suggested that the amniotic membrane inlay filling may aid graft survival.

In all cases, the vascularization into the graft developed successfully, although the previously mentioned four cases required extended periods of time (9-12 weeks). The common findings in the four cases, that is, that avascular areas surrounding the scleral calcific plaques were large, may explain why it took more time for vascularization to occur. In case 4 , it took only 3 days for vascularization, which was the fastest among the patients. The well-vascularized normal conjunctival tissue was located near the scleral calcific plaque, which may be the reason. Interestingly, the area of the new vascular network formation tended to be larger than the area previously occupied by calcific plaques in all cases. In fact, I found that the newly vascularized area was enlarged during follow-up. The vascular network also supplied ischaemic areas adjacent to the calcific plaque. There might be an angiogenic effect in the amniotic membrane in addition to the well-known anti-angiogenic action, ${ }^{20-22}$ as Faulk et $a l^{23}$ suggested. 


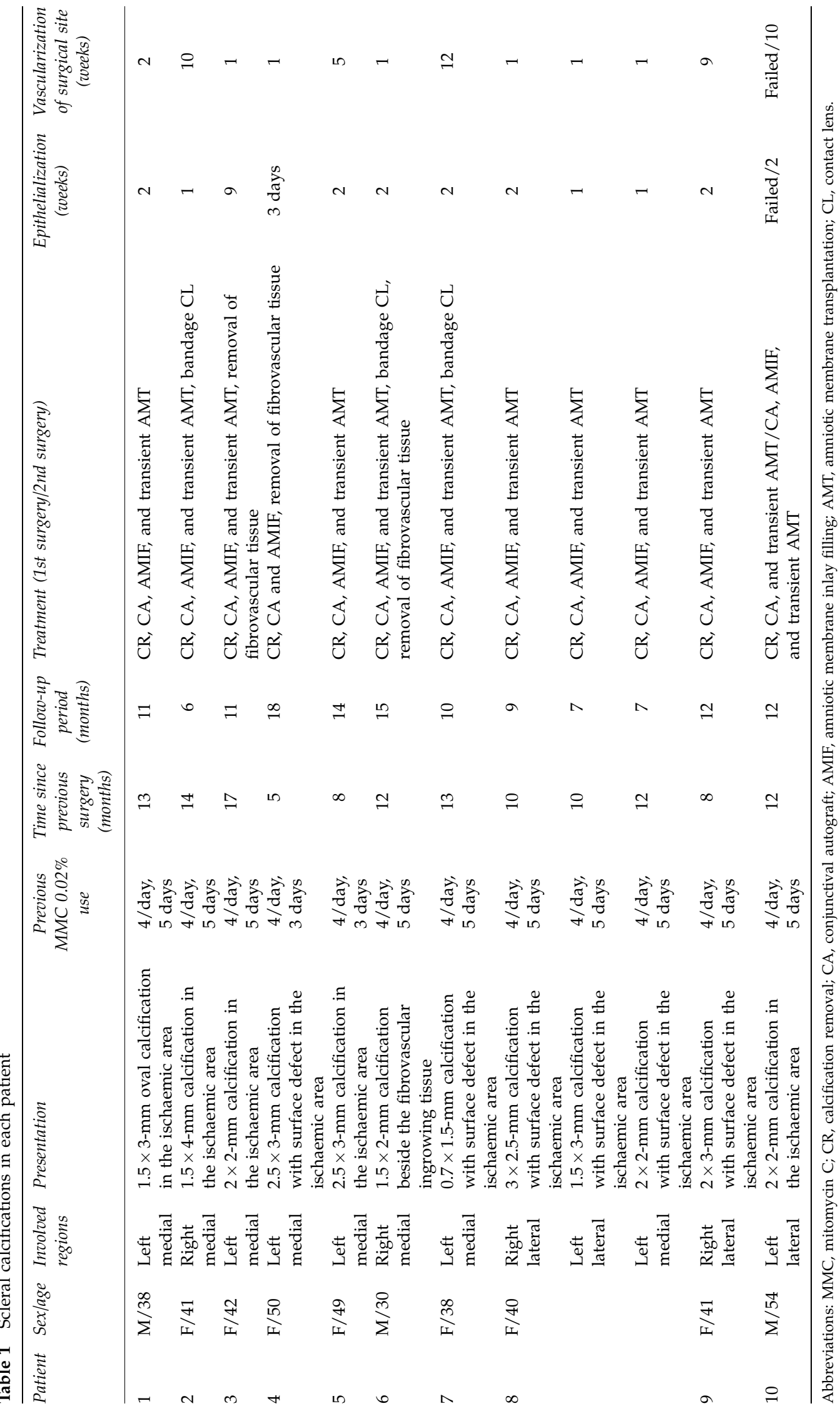



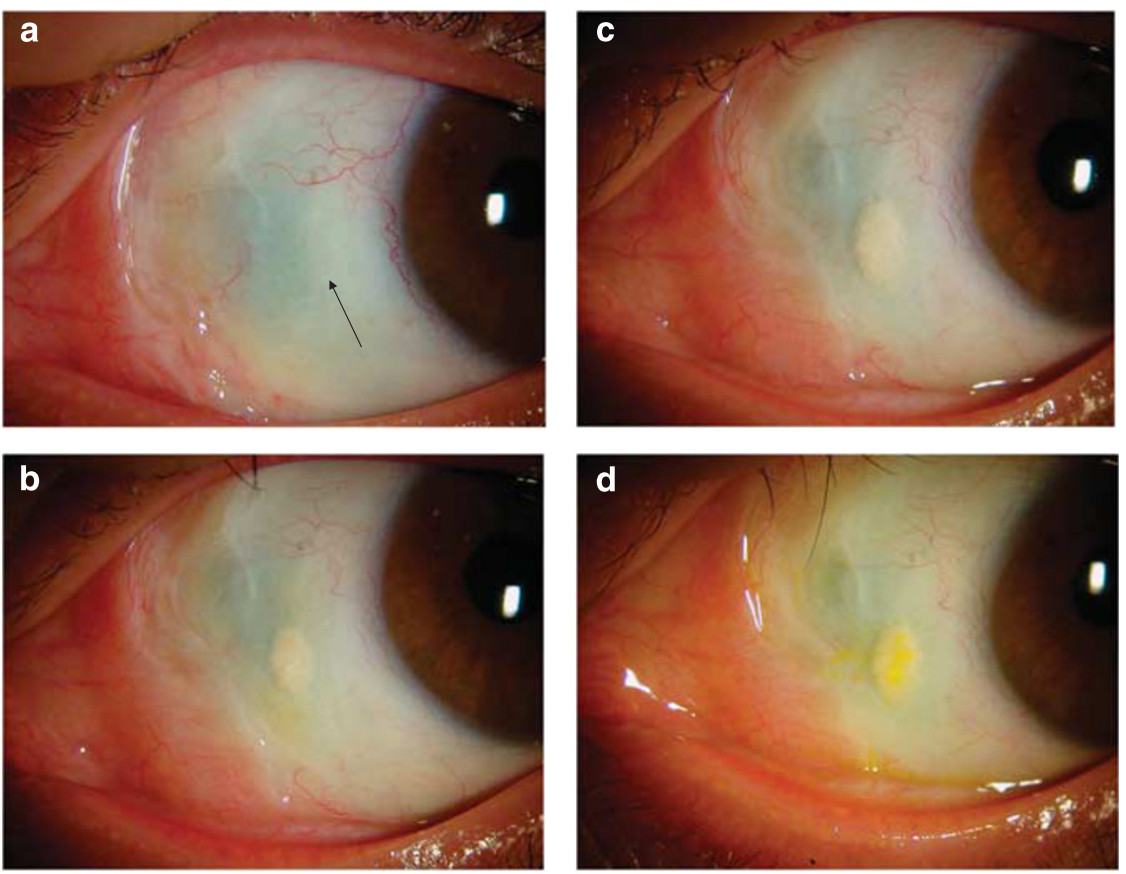

Figure 1 Development and progression of scleral calcification in case 1. (a) Three months after the regional conjunctivectomy was performed to remove pinguecula, an area without vascularization has developed on the medial side of the left eye (arrow). (b) Nine months later, a white oval calcific plaque has developed in the ischaemic scleral area. (c) The scleral plaque increased in size 4 months later. (d) A surface defect is seen with fluorescein staining over the calcific plaque.

Fast epithelial wound healing was achieved in the current study. A duration of 1 or 2 weeks was sufficient for graft epithelialization, except in case 3 and the first surgery in case 10 . Temporary amniotic membrane transplantation was used as a biological bandage, as reported. ${ }^{19,24,25}$ In case 3, early exposure of the grafted site occurred 1 week postoperatively because of a partial detachment of the transiently transplanted amniotic membrane, which has a role in protecting the surgical site. The detached amnion was supposed to be in place for 2 weeks. This early detachment of the transient amnion may prevent adequate protection of the graft for a sufficiently long time and, thus, may result in delayed epithelialization. Temporary amniotic membrane transplantation and use of a 19-mm oversized contact lens to protect the graft may be beneficial. The characteristics that promote epithelialization of the amniotic membrane itself also may contribute. ${ }^{26-28}$ The speed with which the wounds healed agreed with those reported previously. $\mathrm{Oh}$ and $\mathrm{Kim}^{29}$ reported that epithelialization of the scleral surface occurred in 1 week when they used amniotic membrane in seven of eight patients with scleromalacia. Hanada et $a l^{30}$ also reported that an average period of 16.5 days was required for complete epithelialization when a multilayered amniotic membrane was used to treat severe corneal and scleral ulcerations.
When compared with scleral or other grafts, this technique is time-saving, relatively simple, and easy for patients because the technique does not require sutures as much as grafts using other materials. The oversized bandage contact lens provides relief postoperatively. A disadvantage of the technique is that it may not be adequate if areas of calcification are large and deep, because the amniotic membrane and the conjunctiva may not provide sufficient structural support.

The pathogenesis of the scleral calcification associated with MMC is unknown. In the current study, I observed that scleral calcification developed initially and progressed because it occurred during follow-up after previous pterygium or pinguecula surgery. Because the calcific plaques developed in an avascular area in almost all cases, development of the avascular ischaemic area is a prerequisite for initial formation of the calcific plaque. The area might lack nutrition and hydration, thus leading to scleral desiccation. Over time, as the scleral calcification grows as much as the avascular ischaemic area allows, the sclera becomes thinner and thinner, which may result in scleromalacia. An immune mechanism may not be involved in this process because no active inflammation was present. This suggests that the scleral calcification without inflammation results from avascular ischaemic damage to the sclera. To the best of my knowledge, this is the first study to report the 


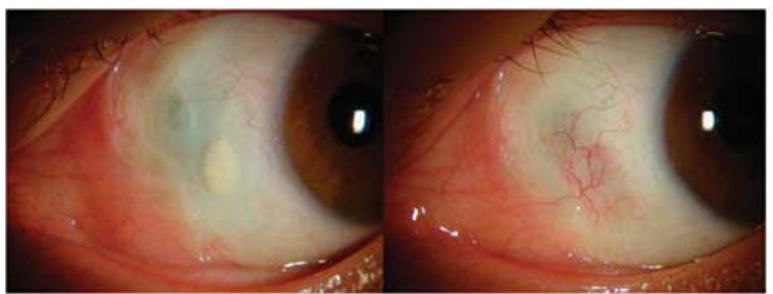

Case 1

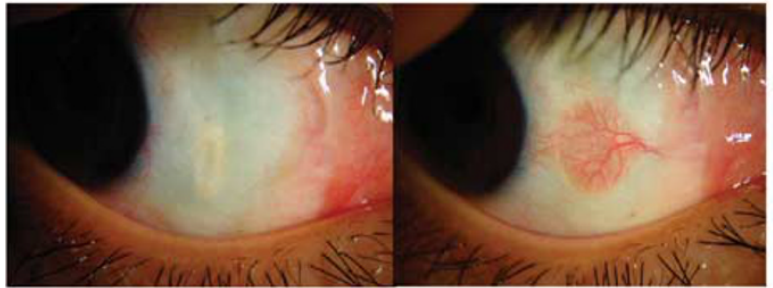

Case 2

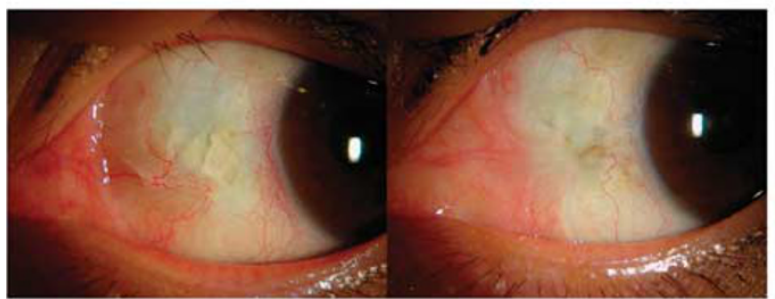

Case 3

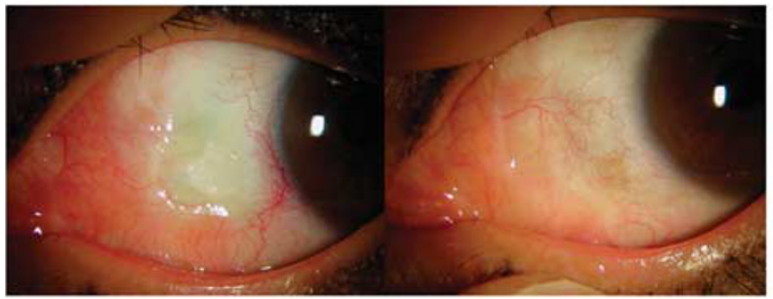

Case 4

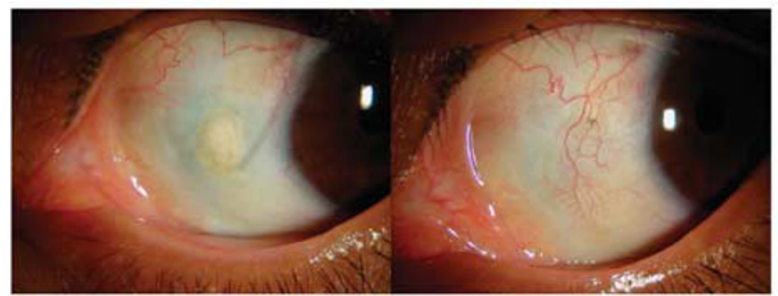

Case5

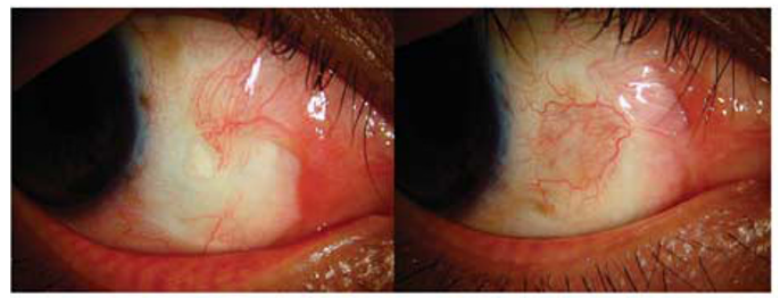

Case 6

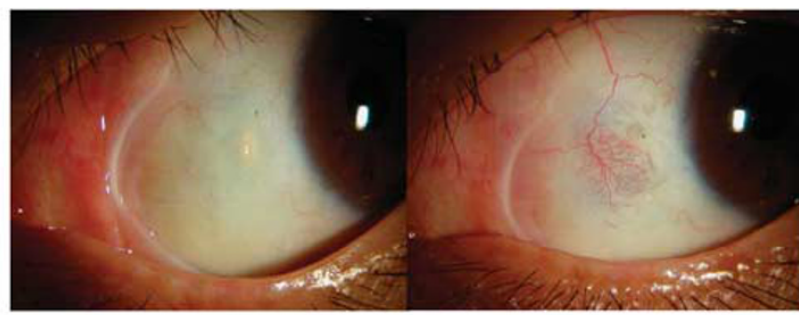

\section{Case 7}

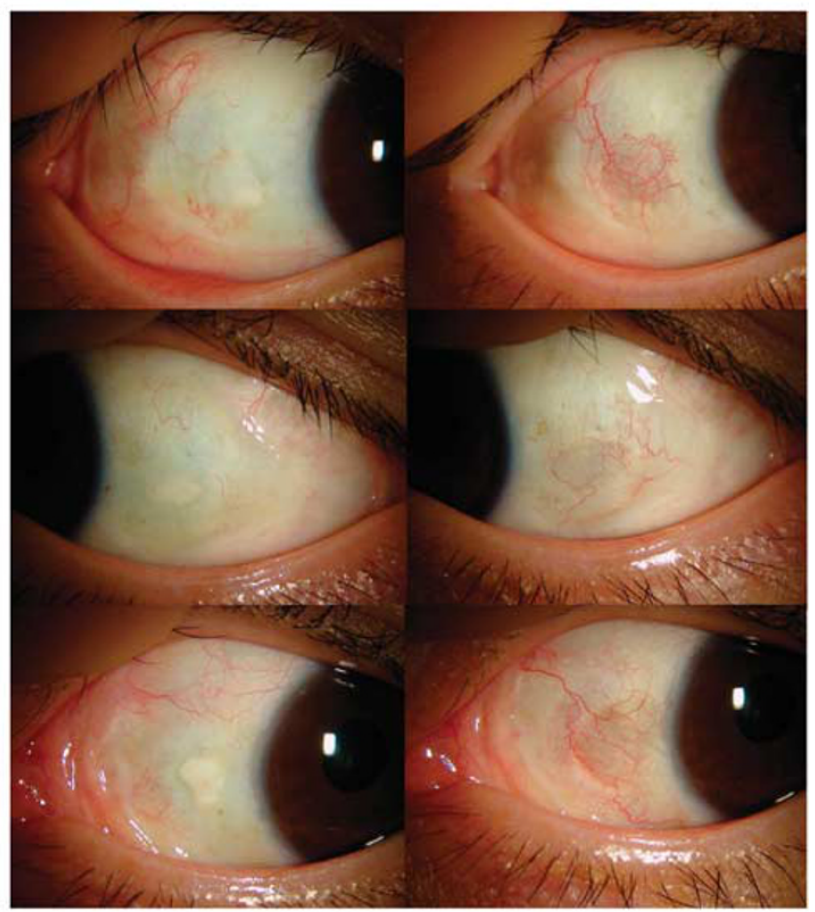

Case 8

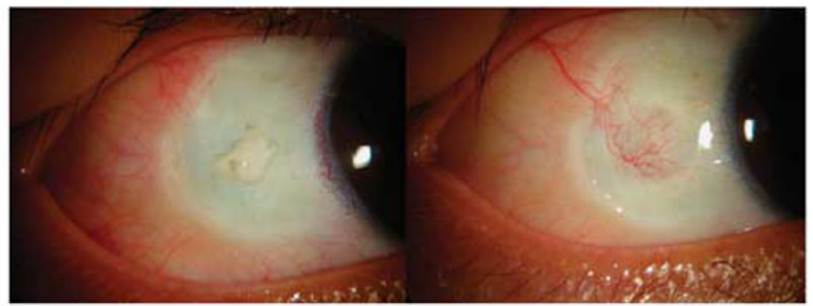

Case 9

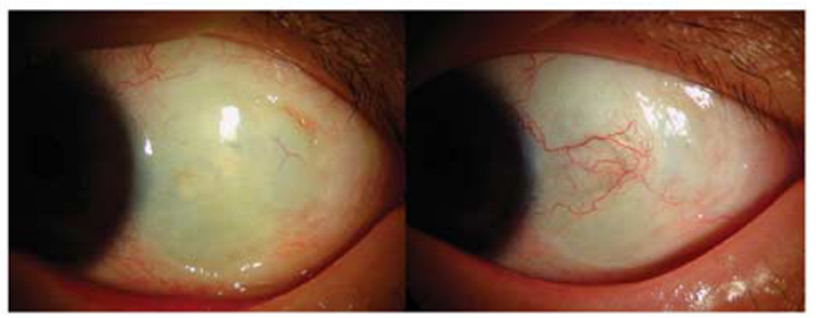

Case 10

Figure 2 Slit-lamp biomicroscopic findings preoperatively (left) and at the final visit postoperatively (right) in the 10 cases. The ocular surface on the final visit is well vascularized and reconstructed. 
manner in which scleral calcification develops and treatment for the early stage of calcification.

In conclusion, removal of scleral calcification, and the combined conjunctival autografting and amniotic membrane inlay filling technique provided revitalization of the necrotic scleral area, and tectonic and cosmetic success was achieved. Calcific plaque formation was identified in the avascular ischaemic areas, and therefore scleral ischaemia may have an important role in development of scleral calcification.

\section{Summary}

What was known before

- Early treatment of scleral calcification has not been extensively studied.

- The exact mechanism of development of scleral calcification is unknown.

What this study adds

- The surgical technique described in this study provided good revitalisation of the necrotic area of scleral calcification. This restoration of the ocular surface may prevent further devastating complications such as infectious scleritis or scleromalacia.

- Scleral ischaemia may induce scleral calcification.

\section{Conflict of interest}

The author declares no conflict of interest.

\section{References}

1 Yamanouchi U. A case of scleral calcification due to mitomycin C instillation after pterygium operation. Folia Ophthalmol Jpn 1978; 29: 1221-1225.

2 Rubinfeld RS, Pfister RR, Stein RM, Foster CS, Martin NF, Stoleru $S$ et al. Serious complications of topical mitomycin-C after pterygium surgery. Ophthalmology 1992; 99: 1647-1654.

3 Dunn JP, Seamone CD, Ostler HB, Nickel BL, Beallo A. Development of scleral ulceration and calcification after pterygium excision and mitomycin therapy. Am J Ophthalmol 1991; 112: 343-344.

4 Okhravi N, Odufuwa B, McCluskey P, Lightman S. Scleritis. Surv Ophthalmol 2005; 50: 351-363.

5 Mauriello JA, Pokorny K. Use of split-thickness dermal grafts to repair corneal and scleral defects - a study of ten patients. Br J Ophthalmol 1993; 77: 327-331.

6 Chechelnitsky M, Mannis MJ, Chu TG. Scleromalacia after retinal detachment surgery. Am J Ophthalmol 1995; 119: 803-804.

7 Raviv T, Greenfield DS, Liebmann JM, Sidoti PA, Ishikawa H, Ritch R. Pericardial patch grafts in glaucoma surgery. J Glaucoma 1998; 1: 27-32.

8 Schein OD. The use of processed pericardial tissue in anterior ocular segment reconstruction. Am J Ophthalmol 1998; 125: 549-552.

9 Melamed S, Ashkenazi I, Belcher III DC, Blumenthal M. Donor scleral graft patching for persistent filtration bleb leak. Ophthalmic Surg 1991; 22: 164-165.
10 Kosmin AS, Wishart PK. A full-thickness scleral graft for the surgical management of a late filtration bleb leak. Ophthalmic Surg Lasers 1997; 28: 461-468.

11 Mistlberger A, Biowski R, Grabner G. Repair of a late-onset filtering bleb leak using a corneal graft shaped with an excimer laser. Ophthalmic Surg Lasers 2001; 32: 428-431.

12 Soong HK, Meyer RF, Wolter JR. Fistula excision and peripheral grafts in the treatment of persistent limbal wound leaks. Ophthalmology 1988; 95: 31-36.

13 Ti SE, Tan DT. Tectonic corneal lamellar grafting for severe scleral melting after pterygium surgery. Ophthalmology 2003; 110: $1126-1136$.

14 Teping C, Reim M. Tenonplasty as a new surgical principle in the early treatment of the most severe chemical eye burns. Klin Monatsbl Augenheilkd 1989; 194: 1-5.

15 Casas VE, Kheirkhah A, Blanco G, Tseng SC. Surgical approach for scleral ischemia and melt. Cornea 2008; 27: 196-201.

16 Mansour AM, Bashshur Z. Surgically induced scleral necrosis. Eye 1999; 13: 723-724.

17 Gregory ME, Weir CR, Ramaesh K. Excision of granulation tissue and free conjunctival autograft in the management of necrotizing scleritis. Cornea 2010; 29: 577-579.

18 Kim B. Regional conjunctivectomy with postoperative mitomycin $\mathrm{C}$ to treat chronic hyperemic conjunctiva. Cornea (in press).

19 Lee SH, Tseng SC. Amniotic membrane transplantation for persistent epithelial defects with ulceration. Am J Ophthalmol 1997; 123: 303-312.

20 Hao Y, Ma DH, Hwang DG. Identification of antiangiogenic and antiinflammatory proteins in human amniotic membrane. Cornea 2000; 19: 348-352.

21 Kim JC, Tseng SCG. The effects on inhibition of corneal neovascularization after human amniotic membrane transplantation in severely damaged rabbit corneas. Korean $J$ Ophthalmol 1995; 9: 32-46.

22 Kobayashi N, Kabuyama Y, Sasaki S, Kato K, Homma Y. Suppression of corneal neovascularization by culture supernatant of human amniotic cells. Cornea 2002; 21: 62-67.

23 Faulk WP, Matthews R, Stevens PJ. Human amnion as an adjunct in wound healing. Lancet 1980; 1: 1156-1158.

24 Kim JS, Kim JC, Na BK. Amniotic membrane patching promotes healing and inhibits proteinase activity on wound healing following acute corneal alkali burn. Exp Eye Res 2000; 70: 329-337.

25 Solomon A, Meller D, Prabhasawat P, John T, Espana EM, Steuhl KP et al. Amniotic membrane grafts for nontraumatic corneal perforations, descemetoceles and deep ulcers. Ophthalmology 2002; 109: 694-703.

26 Guo M, Grinnell F. Basement membrane and human epidermal differentiation in vitro. J Invest Dermatol 1989; 93: 372-378.

27 Meller D, Pires RTF, Tseng SC. Ex vivo preservation and expansion of human limbal epithelial cells by amniotic membrane. Invest Ophthalmol Vis Sci 1999; 40: 329.

28 Khodadoust AA, Silverstein AM, Kenyon DR, Dowling JE. Adhesion of regenerating corneal epithelium. The role of basement membrane. Am J Ophthalmol 1968; 65: 339-348.

$29 \mathrm{Oh} \mathrm{JH}, \mathrm{Kim}$ JC. Repair of scleromalacia using preserved scleral graft with amniotic membrane transplantation. Cornea 2003; 22: 288-293.

30 Hanada K, Shimazaki J, Shimmura S, Tsubota K. Multilayered amniotic membrane transplantation for severe ulceration of the cornea and sclera. Am J Ophthalmol 2001; 131: $324-331$. 